\title{
The Influence of Glass Content on the Hydraulic Conductance and Tubule Occlusion of Novel Bioactive GlassToothpastes
}

\author{
Sing Fong Tie, Robert Hill and David G. Gillam* \\ Institute of Dentistry, Barts and the London School of Medicine and Dentistry, Queen Mary University of London, UK
}

${ }^{\star}$ Corresponding author: David G Gillam, Oral Bioengineering, Institute of Dentistry, Barts and the London, School of Medicine and Dentistry, Queen Mary University London, London E1 2AD, UK

Received: August 12, 2020; Accepted: August 17, 2020; Published: August 24, 2020

\begin{abstract}
Bioactive glasses are widely used as additives in remineralising toothpastes for treating dentine hypersensitivity, which is associated with dentinal fluid flow within exposed dentinal tubules. This study investigates the weight percentage of a fluoride containing bioactive glass in a toothpaste formulation on tubule occlusion and fluid flow of mid coronal dentine discs. Tests were performed after brushing, after immersion in artificial saliva and after a citric acid challenge.
\end{abstract}

There was a statisticallly significant reduction in fluid flow after application of all the toothpastes but the fluid flow reduction was not statistically significantly above a $5 \%$ loading of bioactive glass in the toothpaste. Immersion In artificial saliva after brushing reduced the fluid flow for all loadings but was not statisticaly significant, whilst an acid challenge increased the fluid flow but was again not statistically significant.

SEM observations of mid coronal sections showed improved occlusion of the dentinal tubules up to a $5 \%$ loading but minimal further improvement for higher loadings.

In conclusion a $5 \%$ loading of bioactive glass is close to optimum for reducing fluid flow and tubule occlusion.

Keywords: Bioactive glass, Glass loading, Hydraulic conductance, Tubular occlusion, Dentine hypersensitivity

\section{Introduction}

Dentine Hypersensitivity (DH) is characterized as a sharp dental pain of short duration caused by the reaction of exposed dentine surfaces to stimuli, typically thermal, evaporative, tactile, osmotic or chemical that cannot be associated to any other dental defect of pathology [1]. It is based on the hydrodynamic theory where stimuli applied onto the exposed dentinal tubules cause fluid movement across the dentine, passing them to the intradental pulpal nerves and thereby initiating a painful sensation [2].

$\mathrm{DH}$ is known to usually impact on the quality of life of affected individuals by instigating painful sensation during eating and drinking hot and cold food and beverages [3]. The prevalence of DH in UK is estimated at $52 \%$ [4]. This figure however drops significantly when the conduct of the study shifted from patients' self-reported questionnaires to clinical examinations by dentists. In clinical studies, the reported prevalence is $2.8 \%$ [5] and $18 \%$ [6] respectively.

The hydrodynamic theory is consistent with the observation that when DH is treated with a tubule-occluding agent, this will result in a reduction in $\mathrm{DH}[7,8]$. Occlusion of exposed dentinal tubules is a widely used strategy for treating $\mathrm{DH}$, and many Over-The-Counter (OTC) toothpastes propose tubule occlusion as their mode of action.
Previously, a bioactive glass (NovaMin ${ }^{\oplus}$, developed originally by NovaMin Technology Inc., Alachua, FL, USA) based on the original 45S5 Bioglass ${ }^{\circledR}$ composition (US Biomaterials Corp. Jacksonville, FL, USA) has been used as a remineralising and occluding ingredient in toothpaste formulations for treating DH [9-17]. This works by precipitating Hydroxycarbonate Apatite (HCA) onto the tooth surface and subsequently occluding the dentinal tubules [12-17]. However, concerns have been expressed over the long-term durability of HCA in the mouth, and formation of Fluorapatite (FAp), rather than HCA is preferable, as it is more resistant to subsequent acid attack and dissolves less readily when exposed to acids (e.g. during consumption of fruit juice and carbonated beverages).

In recent years solid state Nuclear Magnetic Resonance Spectroscopy has been used to understand bioactive glass structure, enabling new bioactive glass compositions to be developed $[18,19]$ with vastly improved bioactivity. Of particular note here are the fluorine containing bioactive glasses developed by Brauer et al. [20,21] and the high phosphate fluorine containing glasses developed by Mneimne et al. [22]. These new glass compositions release fluoride in addition to calcium and phosphate and form fluorapatite and have recently been developed specifically for toothpastes [23-31]. 
The objective of this paper is to investigate the ability of one of these new fluorine containing glasses to occlude dentinal tubules and to reduce fluid flow as a function of the amount of glass in the paste immediately after treatment, after immersion in saliva and after an acid challenge.

\section{Materials and Methods}

The materials and methods consisted of two stages. The first stage involved the preparation of the raw materials to be used for the study whereas the second stage comprised the experimental design, which covered the conduct of the study.

\section{Collection of Teeth}

A total of 108 extracted, caries-free human molars were collected from patients attending the walk-in clinic at Tanah Puteh Dental Clinic, Malaysia from May 2016 to August 2016. In accordance with local ethics in the Malaysian clinic verbal consent was obtained from patients who required extraction of their teeth. Following extraction, the teeth were washed and stored in Listerine (Listerine Original) mouthwash solution at room temperature. The collected teeth were then brought to London in September 2016 by SFT under strict health and safety guidelines as required by QMUL. On arrival in the UK laboratory the teeth transferred to a $70 \%$ Ethanol solution until the commencement of the study.

\section{Preparation of Mid Coronal Dentine Sections}

All the collected human molar teeth were prepared into dentine discs of $1.3 \mathrm{~mm}$ thickness using an automatic precision cutting machine (Struers Accutom 5, Denmark). The dentine discs were then ground using a Kemet 4 machine (Kemet Maidstone Kent ME15 9NJ UK) followed by polishing with three different silicon carbide papers in a descending order of abrasive coarseness, starting from carbide paper grade P600, P1000 to P2500. The polishing was considered complete when the discs were polished to the thickness of $1.0 \mathrm{~mm}$. The thickness of the discs was monitored constantly using a digital micrometer to avoid over polishing.

\section{Etching of Dentine Sections}

The etching of dentine discs was only performed just before the discs were to be used for the experimental steps. It was undertaken by dipping the discs into $6 \% \mathrm{w} / \mathrm{w}$ citric acid solution for 30 seconds. The purpose of etching was to remove any smear layer on the discs, thereby opening up the tubules [32]. The discs were then ultrasonicated with deionized water in an ultrasonic bath for 30 seconds to remove any residual acid.

\section{Preparation of Artificial Saliva}

The artificial saliva is based on a formulation first proposed by Featherstone et al. [32] and consists of 2.24 grams of $\mathrm{KCl}, 1.36$ grams of $\mathrm{KH}_{2} \mathrm{PO}_{4}, 0.76$ grams $\mathrm{NaCl}, 0.44$ grams of $\mathrm{CaCl}_{2} .2 \mathrm{H}_{2} \mathrm{O}, 2.2$ grams of porcine Mucin and 0.2 grams of $\mathrm{NaN}_{3}$ (all Sigma-Aldrich, UK) were mixed with 800 grams of deionized water in a 1 litre volumetric flask. The mixture was stirred using a magnetic hotplate stirrer for 30 minutes until all reagents were fully dissolved. The mixture's $\mathrm{pH}$ was then adjusted to 6.5 at room temperature using a $\mathrm{pH}$ meter (Oakton, Netherlands) by adding $0.5 \mathrm{M}$ of $\mathrm{KOH}$ sequentially until the desired
$\mathrm{pH}$ was obtained. Separately, $0.5 \mathrm{M}$ of $\mathrm{KOH}$ was prepared beforehand by mixing 1.40 grams of $\mathrm{KOH}$ (Sigma-Aldrich, $\mathrm{UK}$ ) in $50 \mathrm{ml}$ deionized water. The final mixture was made up with deionized water to 1 litre. The produced artificial saliva solution was kept in a fridge set at $5{ }^{\circ} \mathrm{C}$ until required and used within 2 weeks of preparation.

\section{Preparation of Bioactive Glass Toothpaste}

The Bioactive Glass BioMinF ${ }^{\oplus}$ was supplied by CDL Ltd Stoke UK and is a fluoride containing bioactive glass. The particle size of the supplied BioMinF $^{\oplus}$ was characterised using a Malvern 3000 Particle Size analyser (Malvern Pananalytical Malvern WR14 1XZ, UK). For comparison a sample of NovaMin ${ }^{\oplus}$ was obtained from 3M (Ceradyne) Seattle USA.

\section{Preparation of the Bioactive Glass Base Paste}

This stage involved the preparation of the base paste first, which was then mixed with different loading of bioactive glass. The base paste was made in the laboratory according to the formulation in US Patent US 2009/0324516 [33] with slight modifications. Components such as bioactive glass, fluoride and flavour were omitted. The composition of the active and inactive ingredients required to produce 100 grams of base paste are listed in Table 1. Each ingredient was weighed separately and then mixed together with a metallic mixing spatula in a $100 \mathrm{ml}$ plastic container. The end product was kept in room temperature until use.

Five 10 grams toothpastes with a different loading of bioactive glass of $0.0 \%, 2.5 \%, 5.0 \%, 7.5 \%, 10.0 \%$ and $15.0 \%$ by weight were fabricated manually by using a mortar and pestle technique. The composition for each toothpaste is represented in Table 2.

To produce a $2.5 \%$ bioactive toothpaste, 9.75 grams of base paste were added to a clean mortar. This was followed by adding 0.25 grams of bioactive glass. The two elements were mixed thoroughly for 150 seconds. The same methods were applied to produce $5.0 \%, 7.5 \%$, $10.0 \%$ and $15.0 \%$ loading of bioactive toothpaste. All the prepared toothpastes were stored in six separate sealed $50 \mathrm{ml}$ plastic bottles until required.

Table 1: Composition of active and inactive ingredients required to produce 100 gram of base paste.

\begin{tabular}{|l|c|c|}
\hline Component & Function & Weight Percentage \\
\hline Glycerol & Humectant & $68.75 \%$ \\
\hline Polyethylene Glycol & Dispersant and to reduce stickiness & $20.83 \%$ \\
\hline K Acesulfame & Sweetener & $0.48 \%$ \\
\hline Polyacrylic Acid & Binder & $0.59 \%$ \\
\hline Titanium Oxide & Whitener & $1.91 \%$ \\
\hline Syloid 63 & Silica & $7.15 \%$ \\
\hline Sodium Dedecyl Sulfate & Surfactant & $1.01 \%$ \\
\hline
\end{tabular}

Table 2: Composition of Bioactive Glass in Various Loading.

\begin{tabular}{|l|c|c|}
\hline \multirow{2}{*}{ Type of Toothpaste } & \multicolumn{2}{|c|}{ Materials incorporated into each toothpaste } \\
\cline { 2 - 3 } & Base Paste $\mathbf{( g )}$ & Bioactive glass $\mathbf{( g )}$ \\
\hline $0.0 \%$ loading & 10.00 & 0.00 \\
\hline $2.5 \%$ loading & 9.75 & 0.25 \\
\hline $5.0 \%$ loading & 9.50 & 0.50 \\
\hline $7.5 \%$ loading & 9.25 & 0.75 \\
\hline $10.0 \%$ loading & 9.00 & 1.00 \\
\hline $15.0 \%$ loading & 8.50 & 1.50 \\
\hline
\end{tabular}


The experimental design involves two parts:

a) Comparing the dentine permeability by measuring hydraulic conductance (Lp).

b) Comparing the occlusive effect of dentinal tubules using SEM.

\section{Hydraulic Conductance Measurement Procedures}

A split chamber device based on the design by Pashley and Galloway [34] was used. The total distance travelled by the air bubble in 240 seconds was designated as the baseline flow rate, which was allocated a value of $100 \%$ permeability.

\section{Measuring the Dentinal Permeability after Treatment with Toothpaste}

0.4 gram of toothpaste was squeezed onto a brush head (Oral-B Floss Action Replacement) and mounted on an electric rechargeable toothbrush (Oral-B Vitality Plus). Without removing the disc from the Pashley cell, it was treated with the toothpaste for 2 minutes. The brush head bristles were applied on to the discs at an inclination of 90 degrees. Immediately after 2 minutes of brushing, the disc was rinsed with deionized water for 10 seconds.

\section{Measuring the Dentinal Permeability Post-treatment with Artificial Saliva}

The dentine disc together with the Pashley cell were immersed in $40 \mathrm{ml}$ of artificial saliva at room temperature for 1 hour and then rinsed with deionized water for 10 seconds. The dentine permeability was then measured again.

\section{Measuring the Dentinal Permeability Post-treatment with Artificial Saliva and an Acid Challenge}

The disc was next immersed in $30 \mathrm{ml}$ of a $6 \%$ citric acid solution for 2 minutes and rinsed with deionized water for 10 seconds. The dentine permeability was then measured for a final time.

A total of 30 dentine discs were used. They were distributed equally into six groups, with each group treated with a different loading of toothpaste. Each toothpaste was dedicated with a specific brush head to avoid contamination with others. Analysis of the dentinal permeability measurement was conducted as follows:

a) Percentage flow reduction after treatment with toothpaste

$$
=\frac{\mathrm{V}_{0}-\mathrm{V}_{1}}{\mathrm{~V}_{0}} \times 100 \%
$$

b) Percentage flow reduction after treatment with toothpaste and immersion in artificial saliva

$$
=\frac{\mathrm{V}_{0}-\mathrm{V}_{2}}{\mathrm{~V}_{0}} \times 100 \%
$$

c) Percentage flow reduction after treatment with toothpaste, immersion in artificial saliva and acid challenge

$$
=\frac{\mathrm{V}_{0}-\mathrm{V}_{3}}{\mathrm{~V}_{0}} \times 100 \%
$$

where $\mathrm{V}_{0}=$ Dentine permeability at baseline (after acid etch)

$\mathrm{V}_{1}$ = Dentine permeability immediately after toothpaste application
$\mathrm{V}_{2}=$ Dentine permeability following immersion in artificial saliva

$\mathrm{V}_{3}=$ Dentine permeability following acid challenge

Scanning Electron Microscopy was conducted on separate samples according to the same protocols used for the dentine permeability measurements. The discs were sputter coated with gold prior to examination and examined in a FEI Inspect-F SEM.

\section{Results}

\section{Particle Size Analysis}

Table 3 gives the D90, D50 and D10 values for the particle size for two commercially available Bioactive Glasses. The particle size of the BioMinF ${ }^{\bullet}$ glass is somewhat smaller than the NovaMin ${ }^{\bullet}$ and is thought to have been optimised for tubule occlusion with a larger proportion of particles being smaller than the size of dentinal tubules.

\section{Hydraulic Conductance}

Figure 1 shows the percentage reduction in hydraulic conductance or fluid flow reduction (FFR) after brushing the toothpaste on to mid coronal dentine discs. There is a small approximately $20 \%$ reduction in hydraulic conductance after applying the $0 \%$ BG toothpaste that may be a result of silica particles in the paste occluding the tubules. This increases to approximately $30 \%$ for the $2.5 \%$ glass loading and to just over $60 \%$ for a $5 \%$ loading of the glass. Above $5 \%$ there is a much more limited reduction in the hydraulic conductance with the $15 \%$ loading giving a $70 \%$ reduction. Above $5 \%$ loading of glass there is no statistical increase in the FFR (Table 4-7). However the FFR is statistically significant up to a $5 \%$ loading of glass.

Table 5 shows the FFR results after immersion in artificial saliva (AS) and then following an acid challenge to mimic the consumption of an acidic drink. In all cases there is an increase in the FFR from $0 \%$ to $2.5 \%$ to $5 \%$ and these differences are statistically significant (Tables 3 and 4). The results mirror the data before immersion in AS.

Table 3: Particle Size of Bioactive Glasses.

\begin{tabular}{|l|c|c|c|}
\hline BioActive Glass & $\begin{array}{c}\text { D90 } \\
(\boldsymbol{\mu m})\end{array}$ & $\begin{array}{c}\text { D50 } \\
(\boldsymbol{\mu m})\end{array}$ & $\begin{array}{c}\text { D10 } \\
(\boldsymbol{\mu m})\end{array}$ \\
\hline BioMinF $^{\infty}$ & 23.00 & 5.92 & 0.62 \\
\hline NovaMin $^{\bullet}$ & 45.55 & 14.47 & 1.77 \\
\hline
\end{tabular}

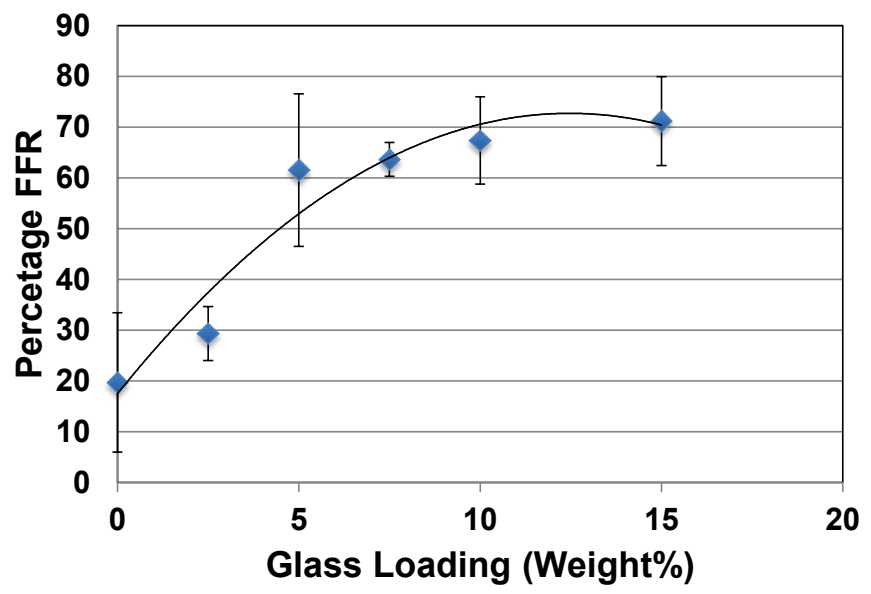

Figure 1: Percentage FFR against BioMinF Loading after Brushing. 
David G. Gillam (2020) The Influence of Glass Content on the Hydraulic Conductance and Tubule Occlusion of Novel Bioactive GlassToothpastes

Above a 5\% loading of BAG in the paste there are no statistically significant increases in the FFR. There was also no significant increase or decrease in the FFR values for any given loading from application to immersion in AS to applying an acid challenge. However, for all glass loadings there is a small decrease in the FFR

Table 4: Statistical Result for Comparison of Percentage Fluid Flow Reduction after Toothpaste Application.

\begin{tabular}{|c|c|c|c|c|c|c|}
\hline \multicolumn{7}{|c|}{ Comparison of Percentage Fluid Flow Reduction after Toothpaste Application } \\
\hline \multirow{2}{*}{ Statistical Data } & \multicolumn{6}{|c|}{ Bioactive Glass Loading } \\
\hline & $0 \%$ & $2.50 \%$ & $5.00 \%$ & $7.50 \%$ & $10.00 \%$ & $15.00 \%$ \\
\hline Sample Mean (in \%), $\mathrm{x}$ & 19.72 & 29.37 & 61.53 & 63.63 & 67.39 & 71.19 \\
\hline Sample sd, s & 13.71 & 5.31 & 15.02 & 3.33 & 8.61 & 8.77 \\
\hline Sample Size, $\mathrm{n}$ & 5 & 5 & 5 & 5 & 5 & 5 \\
\hline Confidence Interval, CI & 1.96 & 1.96 & 1.96 & 1.96 & 1.96 & 1.96 \\
\hline Margin of Error & 12.02 & 4.65 & 13.16 & 2.92 & 7.55 & 7.69 \\
\hline Upper Bound & 31.73 & 34.02 & 74.69 & 66.55 & 74.94 & 78.88 \\
\hline \multirow[t]{2}{*}{ Lower Bound } & 7.70 & 24.72 & 48.36 & 60.70 & 59.84 & 63.51 \\
\hline & \multicolumn{6}{|c|}{ Paired t-test Comparing Each Group $(\mathrm{P}<0.05)$} \\
\hline T-test comparing to $0 \%$ & & 0.128529 & 0.000693 & 0.001276 & 0.002087 & 0.0022316 \\
\hline T-test comparing to $2.5 \%$ & & & 0.004738 & 0.000264 & 0.000104 & $3.269 \mathrm{E}-05$ \\
\hline T-test comparing to $5.0 \%$ & & & & 0.386578 & 0.238683 & 0.1454652 \\
\hline T-test comparing to $7.5 \%$ & & & & & 0.246252 & 0.0712444 \\
\hline T-test comparing to $10.0 \%$ & & & & & & 0.2436429 \\
\hline
\end{tabular}

Table 5: Percentage FFR in Comparison to the Baseline Control after treatment with the toothpaste after treatment with the toothpaste and immersion in artificial saliva (AS) and then with an acid challenge.

\begin{tabular}{|l|c|c|c|}
\hline \multirow{2}{*}{$\begin{array}{l}\text { Bioactive } \\
\text { Loading }\end{array}$} & \multicolumn{3}{|c|}{ Percentage FFR in Comparison with Baseline/Control (\%) } \\
\cline { 2 - 4 } & $\begin{array}{c}\text { After treatment } \\
\text { with toothpaste }\end{array}$ & $\begin{array}{c}\text { After treatment } \\
\text { with toothpaste } \\
\text { and immersion in } \\
\text { artificial saliva }\end{array}$ & $\begin{array}{c}\text { After treatment with } \\
\text { toothpaste, immersion } \\
\text { in artificial saliva and } \\
\text { acid challenge }\end{array}$ \\
\hline $0.0 \%$ & 19.72 & 22.29 & 16.11 \\
\hline $2.5 \%$ & 29.37 & 33.97 & 26.57 \\
\hline $5.0 \%$ & 61.53 & 63.83 & 49.02 \\
\hline $7.5 \%$ & 63.63 & 64.96 & 56.63 \\
\hline $10.0 \%$ & 67.39 & 66.29 & 55.53 \\
\hline $15.0 \%$ & 71.19 & 71.53 & 65.26 \\
\hline
\end{tabular}

Table 6: Statistical Result for Comparison of Percentage Fluid Flow Reduction after Toothpaste Application and Saliva Immersion.

\begin{tabular}{|c|c|c|c|c|c|c|}
\hline \multicolumn{7}{|c|}{$\begin{array}{l}\text { Comparison of Percentage Fluid Flow Reduction after Toothpaste Application and } \\
\text { Saliva Immersion }\end{array}$} \\
\hline \multirow{2}{*}{ Statistical Data } & \multicolumn{6}{|c|}{$\begin{array}{l}\text { Bioactive Glass Loading } \\
\end{array}$} \\
\hline & $\mathbf{0 \%}$ & $2.50 \%$ & $5.00 \%$ & $7.50 \%$ & $10.00 \%$ & $15.00 \%$ \\
\hline Sample Mean (in \%), $\mathrm{x}$ & 22.29 & 33.97 & 63.83 & 64.96 & 66.29 & 71.53 \\
\hline Sample sd, s & 17.14 & 5.58 & 18.70 & 3.85 & 6.04 & 13.59 \\
\hline Sample Size, $\mathrm{n}$ & 5 & 5 & 5 & 5 & 5 & 5 \\
\hline Confidence Interval, CI & 1.96 & 1.96 & 1.96 & 1.96 & 1.96 & 1.96 \\
\hline Margin of Error & 15.03 & 4.89 & 16.39 & 3.38 & 5.29 & 11.91 \\
\hline Upper Bound & 37.32 & 38.86 & 80.22 & 68.34 & 71.58 & 83.44 \\
\hline \multirow[t]{2}{*}{ Lower Bound } & 7.27 & 29.07 & 47.44 & 61.58 & 61.00 & 59.62 \\
\hline & \multicolumn{6}{|c|}{ Paired t-test Comparing Each Group $(\mathrm{p}<0.05)$} \\
\hline T-test comparing to $0 \%$ & & 0.1560 & 0.0011 & 0.001758 & 0.003325 & 0.0073734 \\
\hline T-test comparing to $2.5 \%$ & & & 0.019915 & 0.000576 & 0.000312 & 0.0009269 \\
\hline T-test comparing to $5.0 \%$ & & & & 0.446936 & 0.389115 & 0.2486045 \\
\hline T-test comparing to $7.5 \%$ & & & & & 0.357676 & 0.182191 \\
\hline $\begin{array}{|ll|}\text { T-test } & \text { comparing } \\
10.0 \% & \text { to } \\
\end{array}$ & & & & & & 0.1308723 \\
\hline
\end{tabular}

following an acid challenge, but this was not statistically significant in paired t-tests in regard to either the brushed or the brushed with AS treatments.

\section{Tubule Occlusion}

The tubule occlusion was followed using scanning electron microscopy of the mid coronal dentine discs. Figure 2 shows the SEMs after brushing with the $0 \%$ BioMinF toothpaste. There is no visual evidence of any tubule occlusion, although there are a small number of fine particles on the treated surfaces. Figure 3 shows the SEMs of the $2.5 \%$ BioMinF toothpaste. There is significant tubule occlusion and a marked reduction in their size. The tubules are more obvious following the acid challenge. For dentine discs treated with $5 \%$ or more

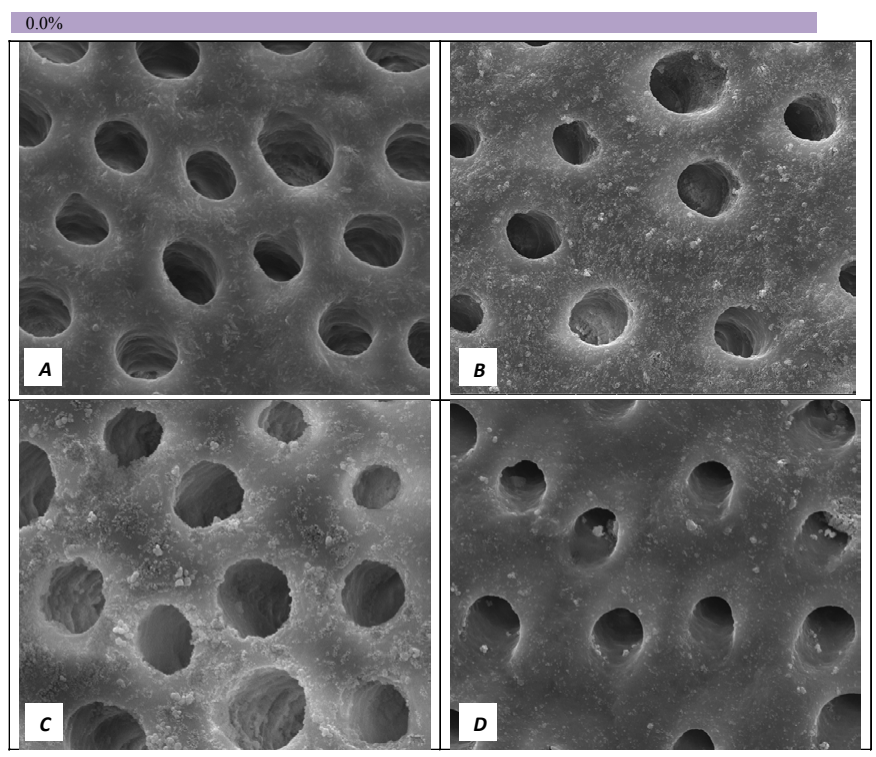

Figure 2: SEM Images seen at x10000 Magnification. (A) Control. (B) After 0.0\% Bioactive Glass Toothpaste Application. (C) Following Saliva Immersion. (D) Following Acid Challenge.

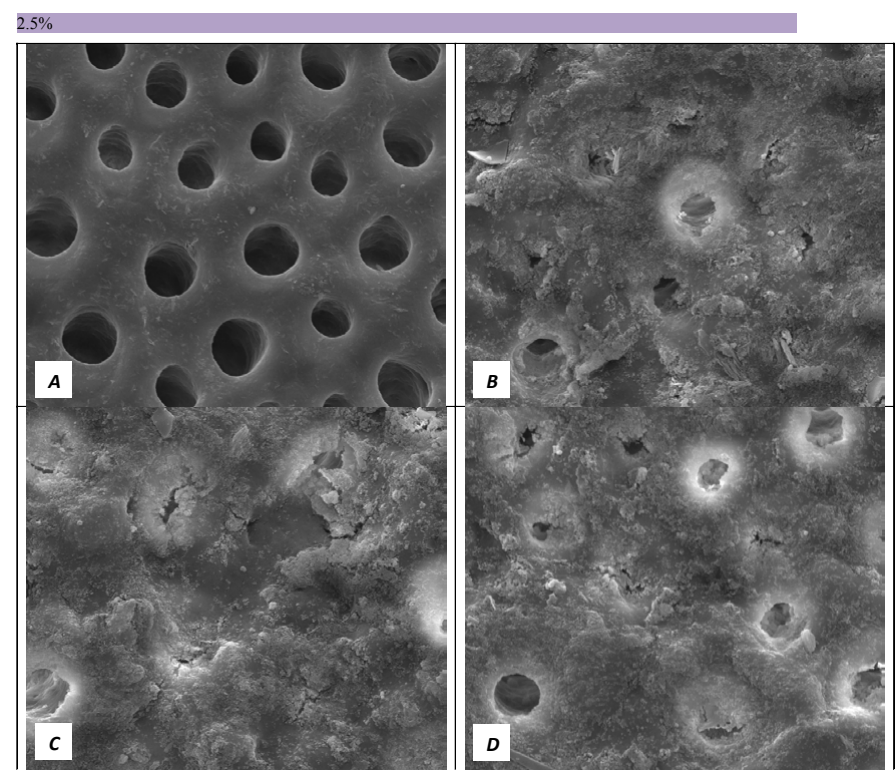

Figure 3: SEM Images seen at x10000 Magnification. (A) Control. (B) After 2.5\% Bioactive Glass Toothpaste Application. (C) Following Saliva Immersion. (D) Following Acid Challenge. 


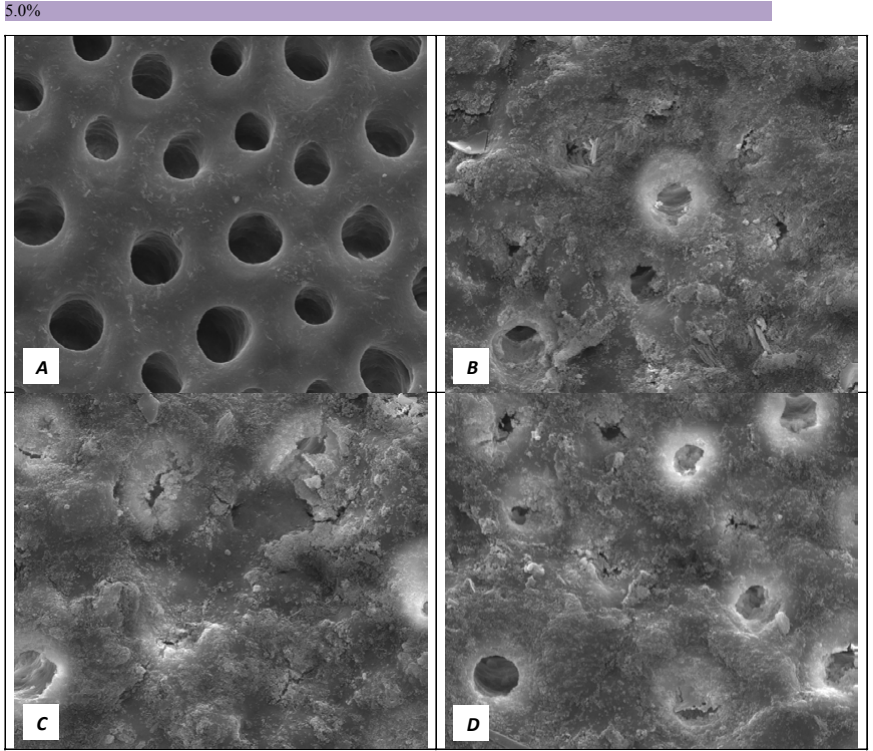

Figure 4: SEM Images seen at X10000 Magnification. (A) Control. (B) After 5.0\% Bioactive Glass Toothpaste Application. (C) Following Saliva Immersion. (D) Following Acid Challenge.

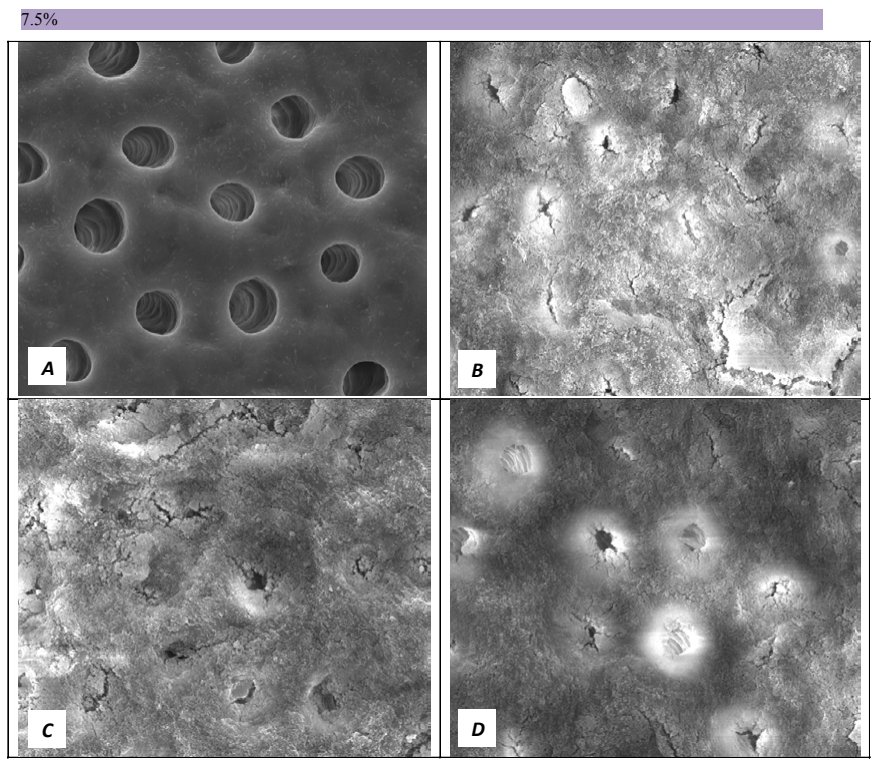

Figure 5: SEM Images seen at x10000 Magnification. (A) Control. (B) After 7.5\% Bioactive Glass Toothpaste Application. (C) Following Saliva Immersion. (D) Following Acid Challenge.

BioMinF loaded toothpastes (Figures 4-8) there is excellent tubule occlusion. There is slight evidence that this might improve slightly after immersion in AS and may deteriorate slightly on exposure to an acid challenge. There may be very slightly better tubule occlusion on increasing the glass loading above $5 \%$.

In general the SEM observations fit well with the hydraulic conductance data and FFR values observed.

\section{Discussion}

In the absence of the glass in the toothpaste formulation there is a small reduction in hydraulic conductance and the FFR is about $20 \%$, There may be some tubule occlusion due to the presence of fine

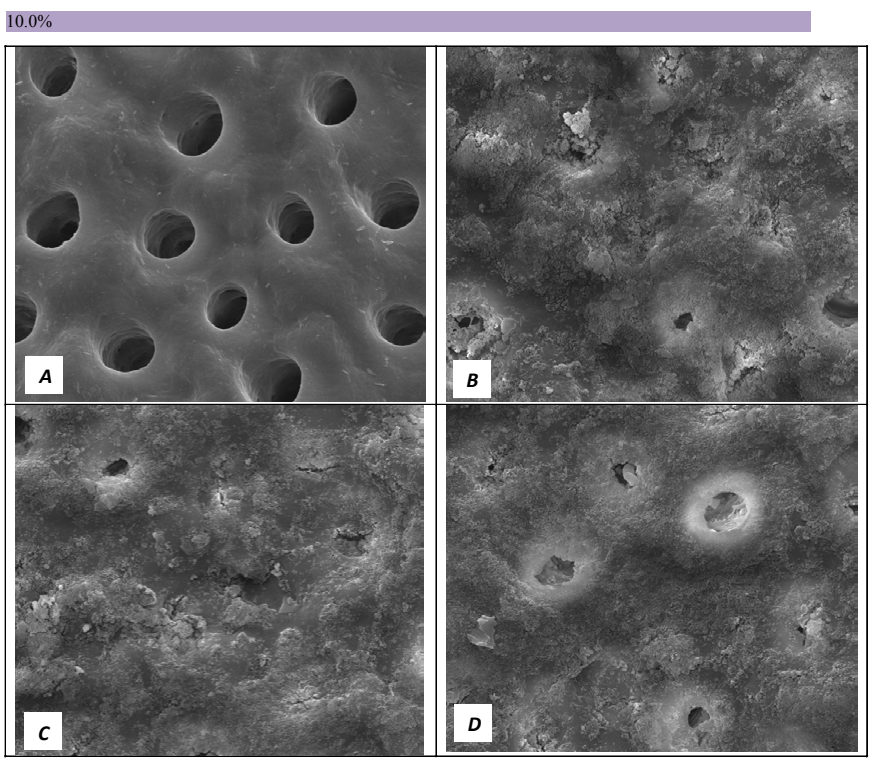

Figure 6: SEM Images seen at x10000 Magnification. (A) Control. (B) After $10.0 \%$ Bioactive Glass Toothpaste Application. (C) Following Saliva Immersion. (D) Following Acid Challenge.

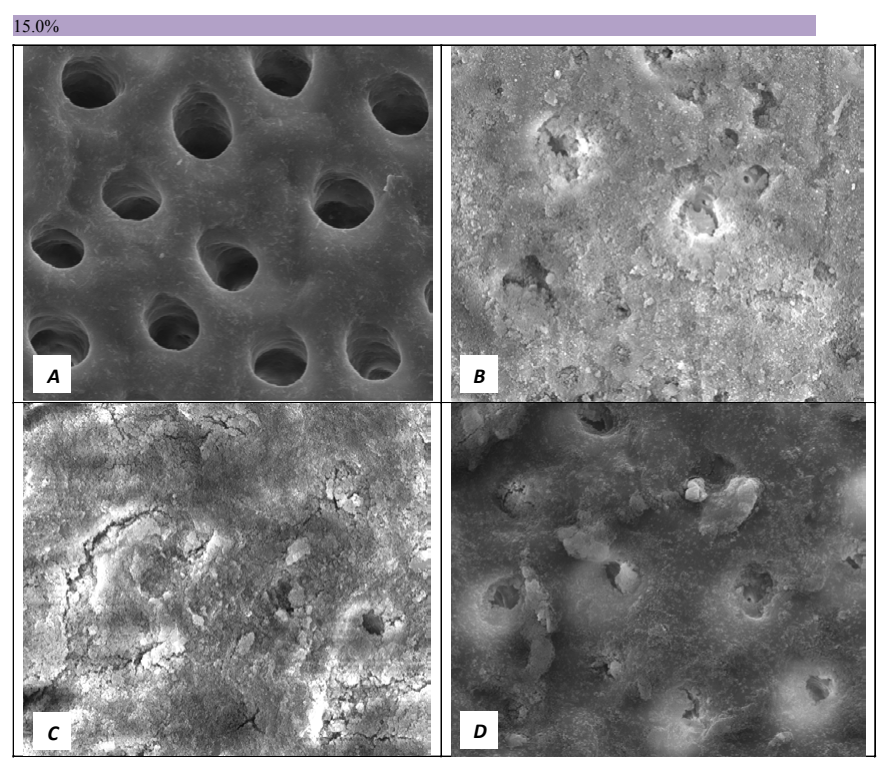

Figure 7: SEM Images seen at x10000 Magnification. (A) Control. (B) After 15.0\% Bioactive Glass Toothpaste Application. (C) Following Saliva Immersion. (D) Following Acid Challenge.

particles in the paste, notably silica and titanium dioxide particles. There is no evidence of any significant tubule occlusion when examined by SEM. On incorporating $2.5 \%$ glass particles into the toothpaste there is a further approximately $10 \%$ increase in FFR. The glass has a D50 of $5.92 \mu \mathrm{m}$ (Table 3) close to the diameter of the larger dentinal tubules and up to $50 \%$ of the glass particles are therefore potentially small enough to enter the dentinal tubules and occlude them. On increasing the loading of glass to $5 \%$ there will be twice as many particles present that are small enough to occlude the dentinal tubules and the FFR increases to more than $60 \%$. Figure 3 shows much greater tubule occlusion for the $5 \%$ glass loading than Figure 2 for the $2.5 \%$ loading. More than $90 \%$ of the tubules are occluded with the $5 \%$ loading. On increasing the bioactive glass content further the There 

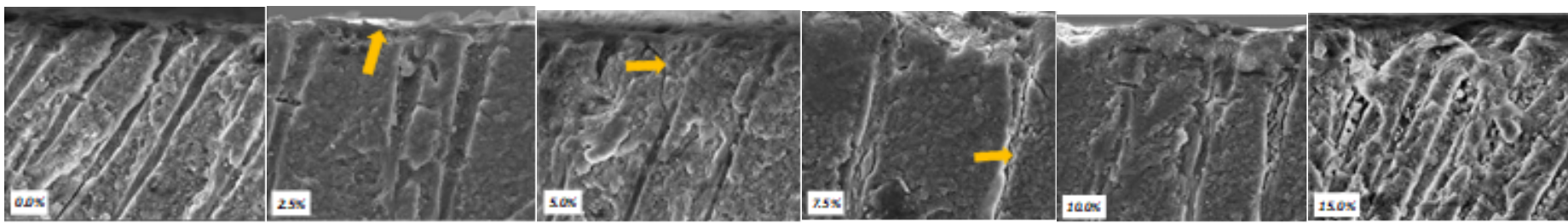

Figure 8: SEM Observation of all bioglass loading(s) following brushing (Cross-Sectional View at x5000 magnification).

At a $0.0 \%$ glass loading, there was no crystal formation at the surface or within the tubules. However, at $2.5 \%$, a thin layer of crystal deposit (arrow) was observed covering the surface. As the bioactive glass loading increased, the crystal deposits were observed to extend deeper into the tubules (arrows). At the 15.0\% bioactive glass loading, large amounts of crystal deposits were observed at the surface of the dentine disc and within the dentinal tubules.

Table 7: Statistical Result for Comparison of Percentage Fluid Flow Reduction after Toothpaste Application, Saliva Immersion and Acid Challenge.

\begin{tabular}{|c|c|c|c|c|c|c|}
\hline \multicolumn{7}{|c|}{$\begin{array}{l}\text { Comparison of Percentage Fluid Fl } \\
\text { Immersion and Acid Challenge }\end{array}$} \\
\hline \multirow{2}{*}{ Statistical Data } & \multicolumn{6}{|c|}{ Bioactive Glass Loading } \\
\hline & $\mathbf{0 \%}$ & $2.50 \%$ & $5.00 \%$ & $7.50 \%$ & $10.00 \%$ & $15.00 \%$ \\
\hline Sample Mean (in \%), $\mathrm{x}$ & 16.11 & 26.57 & 49.02 & 56.63 & 55.53 & 65.26 \\
\hline Sample sd, s & 14.39 & 3.63 & 20.55 & 5.17 & 15.04 & 10.75 \\
\hline Sample Size, $\mathrm{n}$ & 5 & 5 & 5 & 5 & 5 & 5 \\
\hline Confidence Interval, CI & 1.96 & 1.96 & 1.96 & 1.96 & 1.96 & 1.96 \\
\hline Margin of Error & 12.61 & 3.18 & 18.01 & 4.53 & 13.18 & 9.42 \\
\hline Upper Bound & 28.72 & 29.75 & 67.03 & 61.16 & 68.71 & 74.69 \\
\hline \multirow[t]{2}{*}{ Lower Bound } & 3.50 & 23.39 & 31.01 & 52.09 & 42.35 & 55.84 \\
\hline & \multicolumn{6}{|c|}{ Paired t-test Comparing Each Group $(\mathrm{P}<0.05)$} \\
\hline T-test comparing to $0 \%$ & & 0.0734 & 0.0087 & 0.0006 & 0.0093 & 0.0035 \\
\hline T-test comparing to $2.5 \%$ & & & 0.0353 & $5.46 \mathrm{E}-05$ & 0.0047 & 0.0005 \\
\hline T-test comparing to $5.0 \%$ & & & & 0.2447 & 0.2922 & 0.0873 \\
\hline T-test comparing to $7.5 \%$ & & & & & 0.4460 & 0.1220 \\
\hline $\begin{array}{lll}\text { T-test } & \text { comparing } & \text { to } \\
10.0 \% & & \end{array}$ & & & & & & 0.0952 \\
\hline
\end{tabular}

were no statistically significant increases in FFR or any observable increase in tubule occlusion in the SEM micrographs. Above 5\% there are probably sufficient numbers of particles smaller than the dentinal tubule diameter to fully occlude the tubules and more glass particles present in the higher loadings do not improve the tubule occlusion.

On immersion in AS all the samples show a small increase in the FFR values, however it is not statistically significant. Bioactive glasses react with physiological solutions to form apatite and in the case of fluoride containing bioactive glasses such as BioMinF $F^{\oplus}$ fluorapatite is formed. Fluorapatite formation is desirable because fluorapatite is much more resistant to acids than hydroxyapatite or calcium carbonate used to occlude tubules in other proprietary dentine hypersensitivity toothpastes. The micrographs are all very similar to the ones before immersion in AS.

On immersing the samples in $6 \%$ citric acid, to mimic the consumption of an acidic drink in the mouth the FFR values all decrease slightly. However statistically there is no significant reduction. The micrographs do show some evidence of more open tubules following the acid treatment, but the effect is small on tubule occlusion. There seems to be evidence that the material of the surface layer and in the entrances of the tubules is being dissolved but that material deeper within the tubules remains, this phenomenon is most marked in with the $2.5 \%$ glass loading. The surfaces of the dentine discs also become noticeably smoother and there is generally a loss of the angular glass particles on the surface that is probably a result of the citric acid dissolving the remaining glass particles. Bingel et al. [35] showed that bioactive glasses dissolve much more rapidly under acidic conditions.

\section{Conclusions}

- A 5\% loading of glass is close to being optimal in terms of its effect on FFR reduction immediately after brushing, after immersion in AS and after an acid challenge.

- There is no statistically significant increase in FFR after immersion in AS and no statistically significant reduction after an acid treatment.

- There is virtually no tubule occlusion for the $0 \%$ glass toothpaste and an obvious increasing tubule occlusion up to $5 \%$ after this there may be a very slight increase in tubule occlusion with glass loading, but the effect is small.

- There is a slight effect of immersion in AS improving tubule occlusion and there may be a slight reduction in tubule occlusion on applying an acid challenge.

- It is possible that a higher loading of glass would be desirable, but this would involve a significant cost increase.

\section{Reference}

1. Dowell P, Addy M (1983) Dentine hypersensitivity--a review. Aetiology, symptoms and theories of pain production. J Clin Periodontol 10: 341-350. [crossref]

2. Brännström M, Åström A (1972) Hydrodynamics of dentine-its possible relationship to dentinal pain. International Dental Journal 22: 219-227. [crossref]

3. Gillam DG (2013) Diagnosis and management of dentine sensitivity. Dental Nursing 9: 320-327.

4. Gillam DG, Seo HS, Bulman JS, Newman HN (1999) 'Perceptions of dentine hypersensitivity in a general practice population. J Oral Rehabil 26: 710-714. [crossref]

5. Rees JS, Addy M (2004) A cross-sectional study of buccal cervical sensitivity in UK general dental practice and a summary review of prevalence studies'. Int J Dent Hyg 2: 64-69. [crossref]

6. Orchardson R, Collins WJ (1987) 'Clinical features of hypersensitive teeth'. Br Dent J 162: 253-256. [crossref]

7. Pashley DH, Meara JA, Kepler EE, Galloway SE, Thompson SM, et al. (1984) 'Dentin permeability. Effects of desensitizing dentifrices in vitro. J Periodontol 55: 522-5. [crossref]

8. Pashley D (1986) Dentin permeability, sensitivity and treatment through tubule occlusion. Journal of Endodontics 12: 465-474. [crossref]

9. Gillam DG, Tang JY, Mordan NJ, Newman HN (2002) The effects of a novel Bioglass dentifrice on dentine sensitivity: a scanning electron microscopy investigation. J Oral Rehabil 29: 305-313. [crossref] 
10. Burwell A, Jennings D, Muscle D, Greenspan DC (2010) NovaMin and dentin hypersensitivity--in vitro evidence of efficacy. J Clin Dent 21: 66-71. [crossref]

11. LaTorre G, Greenspan DC (2010) The role of ionic release from NovaMin (calcium sodium phosphosilicate) in tubule occlusion: an exploratory in vitro study using radio-labeled isotopes. J Clin Dent 21: 72-76. [crossref]

12. Litkowski L, Greenspan DC (2010) A clinical study of the effect of calcium sodium phosphosilicate on dentin hypersensitivity--proof of principle. J Clin Dent 21: 77-81. [crossref]

13. Salian S, Thakur S, Kulkarni S, LaTorre G (2010) A randomized controlled clinical study evaluating the efficacy of two desensitizing dentifrices. J Clin Dent 21: 82-87. [crossref]

14. Kanwal N, Brauer DS, Earl J, Wilson RM, Karpukhina N. and Hill R.G (2018) "Invitro apatite formation capacity of a bioactive glass - containing toothpaste" J Dent 68: 51-58. [crossref]

15. Greenspan DC (2010) NovaMin and tooth sensitivity-an overview. J Clin Dent 21: 61-65. [crossref]

16. Earl JS, Ward MB, Langford RM (2010) Investigation of dentinal tubule occlusion using FIB-SEM milling and EDX. J Clin Dent 21: 37-41 [crossref]

17. Earl JS, Topping N, Elle J, Langford RM, Greenspan DC (2011) Physical and chemical characterization of the surface layers formed on dentin following treatment with a fluoridated toothpaste containing NovaMin. J Clin Dent 22: 68-73 [crossref]

18. Elgayar I, Aliev AE, Boccaccini AR, Hill RG (2005) Structural analysis of bioactive glasses. Journal of Non-Crystalline Solids 351: 173-183. [crossref]

19. O'Donnell MD, Watts SJ, Hill RG, Law RV (2009) The effect of phosphate content on the bioactivity of soda-lime-phosphosilicate glasses. Journal of Materials Science: Materials in Medicine 20: 1611-1618. [crossref]

20. Brauer DS, Karpukhina N, Law RV, Hill RG (2009) Structure of fluoride-containing bioactive glasses. Journal of Materials Chemistry 19: 5629-5636.

21. Brauer DS, Karpukhina N, O’Donnell M, Law RV, Hill RG (2010) "Fluoride containing bioactive glasses: Effect of glass design and structure on degradation, $\mathrm{pH}$ and apatite formation in simulated body fluid" Acta Biomaterialia 6: 3275-3282.

22. Mneimne M, Hill RG, Bushby AJ and Brauer DS (2011) "High phosphate content significantly increases apatite formation of fluoride-containing bioactive glasses". Acta Biomaterialia 7: 1827-1834. [crossref]

23. Lynch E, Brauer DS, Karpukhina N, Gillam DG and Hill RG (2012) "Multicomponent bioactive glasses of varying fluoride content for treating dentin hypersensitivity". Dental Materials 18: 168-178.
24. Imran Farooq, Imran Alam Moheet, Emad AlShwaimi (2015) In vitro dentin tubule occlusion and remineralization competence of various toothpastes Archives of Oral Biology 60: 1246-1253. [crossref]

25. Patel VR, Shettar L, Thakuri S, Gillam D, Kamal DN (2019) A randomised clinical trial on the efficacy of $5 \%$ fluorocalcium phosphosilicate-containing novel bioactive glass toothpaste. J Oral Rehabil 46: 1121-1126. [crossref]

26. Luiza Pereira Dias da Cruz, Robert G Hill, Xiaojing Chen, David G. Gillam (2018) Dentine Tubule Occlusion by Novel Bioactive Glass-Based Toothpastes. International Journal of Dentistry. [crossref]

27. Shalini D Aggarwal, Anamika Borkar, Nikhil Borse, Aneesha Acharya (2019) Comparative evaluation of fluoro calcium phosphosilicate, calcium sodium phosphosilicate, and strontium chloride hexahydrate containing dentifrice for the treatment of dentin hypersensitivity: A randomized single-blind study. Journal of International Oral Health 11: 404-411.

28. Ella A Naumova, Moritz Staiger, Ouafaa Kouji, Jakov Modri, Thessa Pierchalla, et al. (2019) Randomized investigation of the bioavailability of fluoride in saliva after administration of sodium fluoride, amine fluoride and fluoride containing bioactive glass dentifrices. BMC Oral Health 19: 119.

29. Shaikh K, Pereira R, Gillam DG, Phad S (2018) Comparative evaluation of desensitizing dentifrices containing BioMin ${ }^{\star}$, Novamin ${ }^{\star}$ and fluoride on dentinal tubule occlusion before and after a citric acid challenge- A scanning electron microscope in-vitro study. J Odontol 2: 105.

30. Saqib Ali, Imran Farooq, Ahmad M Al-Thobity, Khalifa S Al-Khalifa, Khalid Alhooshani and Salvatore Sauro (2019) An in-vitro evaluation of fluoride content and enamel remineralization potential of two toothpastes containing different bioactive glasses. Bio-Medical Materials and Engineering 30: 487-496. [crossref]

31. Farooq, I, Majeed A, Al Shwaimi, E, Almasc K (2019) Efficacy of a Novel fluoride Containing Bioactive Glass Based Dentifrice in Remineralising Artificially Induced Demineralisation in Human Enamel. Fluoride 52: 447-455.

32. Matos AB, Palma RG, Saraceni CHC, Matson E (1997) Effects of Acid Etching on Dentin Surface: SEM Morphological Study Braz Dent J 8: 35-41. [crossref]

33. Muscle DP, Burwell KA, La Torre G. Composition and methods for enhancing fluoride uptake using bioactive glass.

34. Pashley DH, and Galloway E (1985)The effects of oxalate treatment on the smear layer of ground surfaces of human dentine. Archives of Oral Biology 30: 731-737. [crossref]

35. Bingel L, Groh D, Karpukhina N, Brauer DS (2015) Influence of dissolution medium pH on ion release and apatite formation of Bioglasss 45S5. Materials Letters 14: 3279-3282.

\section{Citation:}

Sing Fong Tie, Robert Hill and David G. Gillam (2020) The Influence of Glass Content on the Hydraulic Conductance and Tubule Occlusion of Novel Bioactive GlassToothpastes. J Dent Maxillofacial Res Volume 3(3): 1-7. 